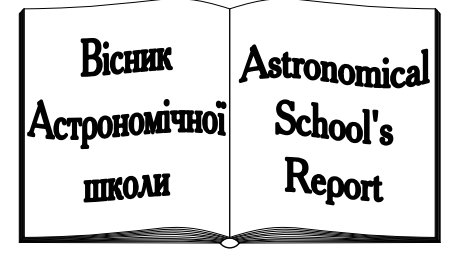

ISSN 1607-2855

Том $\mathbf{1} \cdot$ № $\mathbf{2} \cdot \mathbf{2 0 0 0} \quad$ C. $12-28$

UDC $521 ; 528$

\title{
On the representation of planet's gravitational and magnetic fields. Planet's radial density profiles
}

\author{
A.N. Marchenko \\ State University "Lviv Polytechnic”, Lviv, Ukraine \\ Basic methods of the representation of planet's gravitational and magnetic fields were considered for time-independent and \\ time-dependent potentials. Parameterization of planet's radial density profile was chosen in Gauss' way as normal law. The \\ latter leads to hydrostatic-adiabatic their models with a good modeling of the global trend of the known piecewise density \\ distributions.
}

О ПРЕДСТАВЛЕНИИ ГРАВИТАЦИОННОГО И МАГНИТНОГО ПОЛЕЙ ПЛАНЕТ. РАДИАЛЬНОЕ РАСПРЕДЕЛЕНИЕ ПЛОТНОСТИ ПЛАНЕТ, Марченко А.Н. - Рассмотрены основные методы описания гравитационного и магнитного поля планеты как в статическом случае, так и для временно-зависимого потенциала. Параметризация распределения плотности планет выбрана в виде нормального закона Гаусса, что приводит к гидростатическиадиабатическим их моделям, хорочо описывающим глобальный тренд известных кусочно-непрерывные распределений.

ПРО ПРЕДСТАВЛЕННЯ ГРАВІТАЦІЙНОГО ТА МАГНІТНОГО ПОЛІВ ПЛАНЕТ. РАДІАЛЬНИЙ РОЗПОДІЛ ГУСТИНИ ПЛАНЕТ, Марченко О.М. - Розглянуто основні методи описання гравітаційного та магнітного полів планети як у статичному випадку, так $і$ для залежного від часу потенціалу. Параметризація розподілу густини планет вибрана у вигляді нормального закону Гауса, щуо приводить до гідростатично-адіабатичних їх моделей, які добре описують глобальний тренд відомих кусково-неперервних розподілів.

\section{INTRODUCTION}

First we start from a short review of fundamentals of the Newtonian potential. It is well-known, that the gravitational potential of a solid body (planet) may be expressed by Newtonian integral

$$
V(P)=G \iiint_{v} \frac{d m}{\ell}=\iiint_{v} \frac{\rho}{\ell} d v,
$$

where $G=6.673 \cdot 10^{-8} \mathrm{~cm}^{2} \mathrm{~g}^{-1} \mathrm{sec}^{-2}$ is the gravitational constant; $\rho$ is the volume density of this body; $d v$ is an element of the volume $v ; d m$ is an element of mass; $\ell$ is the distance between the mass element $d m=\rho d v$ and the attracted point $P$ with unit mass. It can be easy verified by differentiating (1) that the force $\mathbf{F}$ of attraction of the current point $P$ is given by

$$
\mathbf{F}=\left(F_{x}, F_{y}, F_{z}\right)=\operatorname{grad} V .
$$

Both the potential (1) and it first derivatives are continuous throughout the whole space (Euclidean threedimensional space $\mathbf{R}^{3}$ ). Besides, the function (1) vanishes at infinity like $1 / \ell$ and it first derivatives at infinity are vanished as $1 / \ell^{2}$ for $\ell \rightarrow \infty$. Therefore the potential $V$ is the so-called regular function at infinity. The second derivatives of $V$ are not continuous throughout the Euclidean space $\mathbf{R}^{\mathbf{3}}$, because the potential (1) satisfies to Poisson's equation 


$$
\Delta V=\frac{\partial^{2} V}{\partial x^{2}}+\frac{\partial^{2} V}{\partial y^{2}}+\frac{\partial^{2} V}{\partial z^{2}}=-4 \pi G \rho .
$$

The density $\rho$ is equal to zero outside the attracting masses (body) and we obtain from (3) the famous Laplace equation

$$
\Delta V=0 \text {. }
$$

Hence, the equation (4) leads to important conclusion that the gravitational potential (1) is the harmonic function outside an attracting body. Inside the Earth $V$ satisfies to Poisson's equation.

Gravity potential of a solid body (planet) is the simple sum (8) of the potentials of the gravitational force $\mathbf{F}$ and the centrifugal force $\Omega$, that is

$$
\text { Щ= } \operatorname{grad} \Omega=\left(\Omega_{x}, \Omega_{y}, \Omega_{z}\right),
$$

where

$$
\Omega=\frac{1}{2} \omega^{2}\left(x^{2}+y^{2}\right),
$$

is the centrifugal potential on the unit mass; $\omega$ is the angular velocity of a planet' rotation. For the components of the centrifugal force $\Omega$ we get from (6):

$$
\Omega_{x}=\omega^{2} x, \quad \Omega_{y}=\omega^{2} y, \quad \Omega_{z}=0 .
$$

Thus, by this definition the gravity potential $W$ may be formed as

$$
W=V+\Omega \text {. }
$$

Note then the gradient of the gravity potential $W$ is the gravity vector $\mathbf{g}$ :

with the following components

$$
\mathbf{g}=\operatorname{grad} W=\left(\frac{\partial W}{\partial x}, \frac{\partial W}{\partial y}, \frac{\partial W}{\partial z}\right),
$$

$$
g_{x}=F_{x}+\Omega_{x}, \quad g_{y}=F_{y}+\Omega_{y}, \quad g_{z}=F_{z}+0 .
$$

This gravity vector is the resultant of the gravitational force (2) and the centrifugal force (5) of a planet' rotation. It can be verified by differentiating (8) that outside a planet the gravity potential (8) satisfies Poisson's equation in the next form

$$
\Delta W=2 \omega^{2} \text {. }
$$

In other words, the gravity potential $\mathrm{W}$ is the non-harmonic function outside the Earth. The magnitude of (9) may be formed as

$$
g=\sqrt{g_{x}^{2}+g_{y}^{2}+g_{z}^{2}} .
$$

Direction of the gravity vector (9) is the so-called direction of the plumb line or vertical.

Thus, in accordance with (2) we may see that the force vector $\mathbf{F}$ or it three components can be replaced by one function $V$ only (Heiskanen and Moritz, 1967). The same results can be found for cases of the Earth and planet's gravity potential (8). For this reason, the determination of planet's gravity fields can be replaced by the determination of their gravity potential. This fact corresponds, in particular, to the main aims of physical geodesy: if the function $W$ is found, we may know all level surfaces including the geoid from the following equation

$$
W(x, y, z)=\text { const } .
$$

Now we recollect that the gravity vector or force vector (9) is normal to the equipotential surface (13) passing through the same point. For this reason the level surfaces (13) and corresponding plumb lines may be used as curvilinear coordinates. For example, the quantities $(\Phi, \Lambda, W$, ) are the so-called natural coordinates (Heiskanen and Moritz, 1967), which are connected with the components of the gravity vector $\mathbf{g}$ as

$$
\Phi=\sin ^{-1}\left(-g_{z} / g\right), \quad \Lambda=\tan ^{-1}\left(g_{y} / g_{x}\right), \quad W=W(x, y, z) .
$$


As a result, the geographical coordinates (the latitude $\Phi$ and the longitude $\Lambda$ ) are entered only by means of the three components of the force vector grad $W$ that corresponds to the physical approach of introducing the threedimensional coordinate system of a planet. Now our following goal is the consideration of various mathematical aspects of the practical representation of time-dependent gravitational and magnetic potentials of the Earth and planets.

\section{A FIRST LOOK AT LAPLACE-LEGANDRE-GAUSS-MAXWELL SPHERICAL HARMONICS}

Starting with fundamental investigations of Laplace, Legendre and Gauss in different areas of the Earth's and planet's study, the classical mathematical description of their gravitational and magnetic fields becomes the expansion into a series of solid spherical harmonics. Now such form of the representation of potential may be considered as one of the best ways for a solution of scientific problems in a global scale. At present, it can be said that the description of the Earth's and planet's potentials by the solid spherical harmonic series of LaplaceLegendre-Gauss kind has the interdisciplinary meaning. Identical representation was developed by Maxwell (1881) and partly by Gauss (1877) as the so-called poles theory or theory of multipole potentials (see, Marchenko, 1998). For this reason we shall call this theory further as Laplace-Legendre-Gauss-Maxwell spherical harmonics applying it for the description of planet's gravitational and magnetic fields.

We start from the expansion (1) of a planet's gravitational potential $V$ into a series of the solid spherical functions $V_{n}$ :

$$
V=\sum_{n=0}^{\infty} V_{n}
$$

Every function $V_{n}$ is connected with the surface spherical function $Y_{n}$, as

$$
V_{n}=Y_{n} / r^{n+1},
$$

where $Y_{n}$ is represented by the linear combination of the zonal, tesseral, and sectorial spherical harmonics

$$
Y_{n}=G M \cdot a^{n} \sum_{m=0}^{n}\left(\bar{C}_{n m} \cos m \lambda+\bar{S}_{n m} \sin m \lambda\right) \bar{P}_{n m}(\cos \vartheta),
$$

and every $Y_{n}$ is characterized by $(2 n+1)$ linear independent harmonic coefficients. In (16) and (17) GM is the product of the gravitational constant on the planet' mass; $a$ is the semimajor axis of its ellipsoid of revolution; $(r, \vartheta, \lambda)$ are the polar coordinates of an external point; $\bar{P}_{n m}(\cos \vartheta)$ are the fully normalized associated Legandre functions; $\bar{C}_{n m}, \bar{S}_{n m}$ are the fully normalized harmonic coefficients.

These coefficients are called also by Stokes' constants, since each of them may be expressed as integral containing the following consistencies

$$
\left.\begin{array}{l}
\bar{C}_{n m} \\
\bar{S}_{n m}
\end{array}\right\}=\frac{1}{(2 n+1) \cdot M \cdot a^{n}} \cdot \iiint_{\text {planet }} \rho\left(r^{\prime}, \vartheta^{\prime}, \lambda^{\prime}\right) \cdot r^{\prime n+2}\left\{\begin{array}{c}
\cos m \lambda^{\prime} \\
\sin m \lambda^{\prime}
\end{array}\right\} \cdot \bar{P}_{n m}\left(\cos \vartheta^{\prime}\right) \cdot \sin \vartheta^{\prime} d r^{\prime} d \vartheta^{\prime} d \lambda^{\prime},
$$

where $r^{\prime n+2}$ is $(n+2)$-degree radius vector of a variable mass element; $\vartheta^{\prime}, \lambda^{\prime}$ are the spherical coordinates of this mass element; $\bar{P}_{n m}\left(\cos \vartheta^{\prime}\right) \cos m \lambda^{\prime}$ or $\bar{P}_{n m}\left(\cos \vartheta^{\prime}\right) \sin m \lambda^{\prime}$ are the corresponding spherical harmonics; $\rho\left(r^{\prime}, \vartheta^{\prime}, \lambda^{\prime}\right)$ is the density at the point $\left(r^{\prime}, \vartheta^{\prime}, \lambda^{\prime}\right)$, (Heiskanen and Moritz, 1967, p.59).

Thus, we have considered the first - classical treatment of the expansion (15)-(18), as the power series expansion, provided by Laplace, Legandre, and Gauss in their fundamental works. Such treatment leads to the well-known ,convergence problem“ of the expansion (15) on the Earth's (planet) surface that, in principle, has only theoretical meaning (Moritz, 1980). For instance, C.F. Gauss has admitted the convergence of (15) on the Earth's surface.

The second - approximate treatment of the expansion (15) is connected with Runge-Krarup theorem (Krarup, 1969; Moritz, 1980), which is formulated usually in the following way. 
Runge - Krarup theorem. Given may be any function $\varphi$ harmonic outside the Earth's surface $\tau$ and regular at infinity as well as a sphere $\sigma_{\mathrm{B}}$ (the so-called Bjerhammar sphere or regularization sphere) located inside the Earth. There exists a sequence of functions $\left\{\varphi_{\mathrm{n}}\right\}$ harmonic outside $\sigma_{\mathrm{B}}$ and regular at infinity converging uniformly to the function $\varphi$ on and outside an auxiliary surface $\tilde{\tau}$ (with finite curvature), which may be arbitrarily close to and surrounding completely the Earth's surface $\tau$.

A detailed discussion of the definition of spaces and the problem of gravity field approximation can be found in (Neyman, 1979; Moritz, 1980, etc.). So, according to the Runge-Krarup theorem the anomalous potential $T=W-U$ (where $U$ is the normal potential) may be found, for instance, among the potentials $\widetilde{T}$, which are regular outside the internal Bjerhammar sphere $\left(\sigma_{B}\right)$. Such approximation profits from the advantageous replacement of the complicated planet' surface $\tau$ by the simple spherical surface $\sigma_{B}$ as boundary, because planet surfaces are close to a spherical surface in the first iteration. In any case, the approximated function $T$ is defined outside $\tau$ on a set $G$ of points and the approximating function $\widetilde{T}$ is defined outside the internal sphere $\sigma_{B}$ on a set $\Sigma$ of points of the three-dimensional Euclidean space $\mathbf{R}^{3}$. Note finally that this theorem may be considered as a three-dimensional analogy of the well-known Weierstrass' theorem on the approximation of any continuos function $y=f(x)$, defined on the interval $[a, b]$, by polynomials.

\section{THE EARTH'S TIME-DEPENDENT GRAVITATIONAL POTENTIAL}

Determination of temporal variations in the Earth's gravitational field becomes one of non-traditional problems in geodesy. As an example, the secular variation $\dot{\bar{C}}_{20}$ in the 2nd degree zonal harmonic coefficient of geopotential was recommended (Bursa, 1992) as a parameter for the aims of IERS (IERS Standards, 1989). Clearly, the possibility of determination of temporal variations of the time-dependent geopotential $V=V(t)$ connects directly with the wide spectrum of various measurements and their level of accuracy. In (Moritz and Mueller, 1987, p. 522-523) we find: "It should be possible to measure temporal variations of the gravity field of the Earth through the deployment of several LAGEOS-type satellites, dense radio-beacon satellites, or longlifetime, drag-free satellite(s) using improved laser (or radio) ranging". Temporal variations of the Earth's gravitational harmonic coefficients are currently estimated today from the satellite data and they are "due to lunisolar tides, meteorological mass redistribution and postglacial rebound of the solid Earth" (Cheng et al., 1989), see also (Yoder et al., 1983; Rubincam, 1984).

At the recent General Assembly of IUGG in the Report of Special Commission 3 on „Fundamental Constants" the more detailed values (see, Table 1) were recommended by (E. Groten, 1999), which were found on the ground of the Earth's gravitational potential model EGM96 by (A. Marchenko and O. Abrikosov, 1999). Further we shall study the description of the time-dependent gravitational field in view of Laplace theory of spherical harmonics and shall consider below two parameterizations of the time-dependent anomalous potential $T(P, t)$ only in the finite interval $[c, d]$ of time $t \in[c, d]$.

On the one hand, some low-degree temporal variations of the Earth's gravity field have a weak dependence on time $(t)$. In the case of the Earth's main magnetic field there exists a more essential dependence on time $t$ both in low and in high frequencies. These two potentials can be studied on the whole as one Newtonian timedependent potential (without zero degree solid spherical harmonics) in view of the well-known measured peculiarities of temporal variations in these fields.

On the other hand, for the Earth's time-dependent potentials we have not such rich theoretical background as in the case of the anomalous time-independent potential. In this area some theoretical considerations regarding to the geodynamical or time-dependent geodetic boundary value problem were investigated (see, for instance, Heck, 1987; Sacerdote and Sanso, 1987; etc.). Nevertheless, various sets of the so-called low-degree secular variations in the Earth's gravitational potential were determined from the satellite data in the frame of liner model. 
Table 1. Parameters of the linear model of the potential of 2nd degree *)

\begin{tabular}{|c|c|c|}
\hline Harmonic coefficient & Value of coefficient $\times 10^{6}$ & Temporal variation $\times 10^{11}\left[\mathrm{yr}^{-1}\right]$ \\
\hline $\bar{C}_{20}$ & -484.165371736 & 1.16275534 \\
$\bar{C}_{21}$ & -0.00018698764 & -0.32 \\
$\bar{S}_{21}$ & 0.00119528012 & 1.62 \\
$\bar{C}_{22}$ & 2.43914352398 & -0.494731439 \\
$\bar{S}_{22}$ & -1.40016683654 & -0.203385232 \\
\hline
\end{tabular}

\subsection{LAPLACE-MAXWELL-TAYLOR PARAMETERIZATION}

First of all we start from Laplace-Maxwell representation of the time-dependent Earth's anomalous potential $T(P, t)$ by a series of solid spherical harmonics which may be written according to (Moritz and Muller, 1987; Marchenko, 1998):

$$
T(P, t)=\frac{G M}{r} \sum_{n=2}^{\infty}\left(\frac{a}{r}\right)^{n} \sum_{m=0}^{n}\left(\bar{C}_{n m}(t) \cos m \lambda+\bar{S}_{n m}(t) \sin m \lambda\right) \bar{P}_{n m}(\cos \vartheta),
$$

where $(r, \vartheta, \lambda)$ are the polar coordinates of an external point $P$; $t$ is current time; $\bar{C}_{n m}(t), \bar{S}_{n m}(t)$ are the fully normalized time-dependent harmonic coefficients of the expansion (19). In the case of only secular variations of harmonic coefficients we apply usually their well-known (truncated) Taylor's form

$$
\left.\begin{array}{l}
\bar{C}_{n m}(t) \\
\bar{S}_{n m}(t)
\end{array}\right\}=\left\{\begin{array}{l}
\bar{C}_{n m} \\
\bar{S}_{n m}
\end{array}\right\}+\left\{\begin{array}{c}
\dot{\bar{C}}_{n m} \\
\dot{\bar{S}}_{n m}
\end{array}\right\} \cdot\left(t-t_{0}\right) \text {, }
$$

where $t$ is any current moment of time; $t_{0}$ is the reference epoch; $\dot{\bar{C}}_{n m}, \dot{\bar{S}}_{n m}$ are the temporal (secular) variations.

Note that the possibility of the representation (19), that is the function $T(P, t)=T(r, \vartheta, \lambda ; t)$, may be obtained as the corollary of the Runge-Krarup theorem. Briefly, because the function $T(P, t)=T(P ; t=$ const $)$ may be considered as an element of the Hilbert space $H_{2}(\Sigma)$ of harmonic, outside the Bjerhammar sphere, functions (in the domain $\Sigma$ ), hence for every $t=$ const from $T(P ; t=c o n s t) \in H_{2}(\Sigma)$, the expansion (19) is correct (Marchenko, 1998). Also, an additional establishment of the similar assertion for the time-dependent gravitational potential $V=V(P, t)$ requires the additional consideration, because the Runge-Krarup theorem was introduced only for an approximation of the time-independent anomalous potential $T=T(P)$. Thus, the relationships (19) and (20) express the time-dependent potential in the form of Laplace-Maxwell (or LaplaceMaxwell-Taylor), since all estimations of secular variations lead in practice to their actual degree $(n \geq 2)$.

\subsection{LAPLACE-MAXWELL-CHEBYSHEV PARAMETERIZATION}

There exists another form of a mathematically homogeneous representation of time-dependent parameters, which was developed especially for a fast solution of some geodynamical direct problems (Marchenko, 1998). This approach based on Chebyshev-Fourier series and was used successfully for the homogeneous description of the next time-dependent phenomena: the Earth's solid and ocean tides, nutation and tidal variation of UT1 as well. This additional revision of traditional theories arises in consequence of the practical solution of the differential equations of satellite motion because the numerical integration of these equations requires the

*) In contrast to the Table 1, EGM96 model includes only non-zero 2nd degree secular variations $\dot{\bar{C}}_{20}, \dot{\bar{C}}_{21}$ and $\dot{\bar{S}}_{21}$. 
computation of force-vector components in any current time $(t)$. Obviously, a mathematically heterogeneous (and cumbersome) model of motion leads in practice to additional time expenses. For instance, by direct applying the IERS recommendations (1989) we come to various mathematical functions, which depend on several time-dependent variables (from 1 up to 6). Practically we may find the following mathematical functions, which were proposed in the IERS Standards: the simple polynomials, Legandre's polynomials, Chebyshev's polynomials, trigonometric functions, special functions, etc. For this reason, all these traditional time-dependent parameters were transformed into a mathematically homogeneous Chebyshev's expansions on a certain time interval. As a result, the coefficients of such expansions were derived in the closed form as various functions of time $t$ or one variable only.

Below we apply this approach to another parameterization of the time-dependent potential $T$ in the form of Laplace-Maxwell-Chebyshev. Briefly, we come to the series

$$
\widetilde{T}(P, z)=\frac{G M}{r} \sum_{n=2}^{\infty}\left(\frac{a}{r}\right)^{n} \sum_{m=0}^{n}\left\{\sum_{k=0}^{\infty}\left(\bar{C}_{n m}^{k} \cos m \lambda+\bar{S}_{n m}^{k} \sin m \lambda\right) \cdot T_{k}(z)\right\} \bar{P}_{n m}(\cos \vartheta),
$$

which may be used especially for the Earth's time-dependent anomalous potential $T(P, t)$ because real studying $T(P, t)$ is defined on the finite interval $[c, d]$ of time $t \in[c, d]$. Note now, the expression (21) can be found firstly by introducing the linear transformation

$$
t=\frac{1}{2}[(d-c) z+(c+d)]
$$

of the interval $[c, d]$ into the standard interval $[-1,1]$. Secondly, let us consider another function $\widetilde{T}(P, z), z \in[-1,1]$ instead of the function $T(P, t), t \in[c, d]$ at every point $P=$ const . This connects mainly with the transformation (22) of the initial coefficients $\bar{C}_{n m}(t), \bar{S}_{n m}(t)$ in the expansion (19). Finally we introduce some new coefficients $\widetilde{C}_{n m}(z), \widetilde{S}_{n m}(z)$ as the functions of "standard time" $z \in[-1,1]$ and write

$$
\left.\begin{array}{c}
\widetilde{C}_{n m}(z) \\
\widetilde{S}_{n m}(z)
\end{array}\right\}=\sum_{k=0}^{\infty}\left\{\begin{array}{l}
\bar{C}_{n m}^{k} \\
\bar{S}_{n m}^{k}
\end{array}\right\} T_{k}(z) \text {. }
$$

These expansions into Chebyshev's polynomials $T_{k}(z)$ are converging uniformly in the interval $[-1,1]$ if the initial coefficients satisfy to the well-known Dine condition. Thus, instead of the expansion of the function $T(P, t), t \in[c, d]$ we come to $(21)$ in the standard interval $[-1,1]$ with the variable $z \in[-1,1]$ defined by $(22)$.

Now we can compare the expansion (20) of $\bar{C}_{n m}(t), \bar{S}_{n m}(t)$ into Taylor's series at the neighborhood of the point $t_{0}$ (by neglecting the terms of order two and higher) with Chebyshev's approximation (23). Returning in (23) to time $t$ (instead of the variable $z$ ) and comparing (20) with (23), as the power series, for first two terms of (20) we find

$$
\begin{aligned}
& \left.\bar{S}_{n m}\right\}=\sum_{k=0}^{\infty}(-1)^{k}\left\{\begin{array}{l}
\bar{C}_{n m}^{2 k} \\
\bar{S}_{n m}^{2 k}
\end{array} \text {, if } t_{0}=(d-c) / 2,\right. \\
& \left.\begin{array}{l}
\dot{\bar{C}}_{n m} \\
\dot{\bar{S}}_{n m}
\end{array}\right\}=\frac{2}{(d-c)} \sum_{k=1}^{\infty}(-1)^{k-1}(2 k-1)\left\{\begin{array}{l}
\bar{C}_{n m}^{2 k-1} \\
\bar{S}_{n m}^{2 k-1}
\end{array} .\right.
\end{aligned}
$$

Thus, we come to an extremely important conclusion. The expression (24) is nothing else, but the timeindependent (stationary or permanent) part of the harmonic coefficients, which are connected with Chebyshev's coefficients of even degrees. The coefficients (24) correspond to the „static“ Earth without any temporal changes in its gravitational potential.

Another relationship (25) admits a remarkable and natural interpretation also. It is nothing else, but the secular variations of potential coefficients, which are connected with the certain sum (25) of Chebyshev's coefficients of odd degrees. 
Finally we note again that variations of spherical harmonic coefficients of 14th tidal waves were derived (Marchenko, 1998) as Chebyshev's coefficients of the expansion (23) in the closed form. The latter, on the one hand, leads to the creation of the Earth's time-dependent/geodynamical model (21) of the geopotential. On the other hand, by applying Chebyshev's coefficients of the even degrees we get from (24) the coefficients of the stationary or permanent deformation of the Earth's. It is easily seen that in the case of the solid Earth's tides this formula (24) leads to the so-called Honkasalo correction. Thus, step by step the proposed study leads to the sequential investigation of the both time-independent (24) and time-dependent (25) consistencies of the Earth's potential and other geodynamical phenomena.

\subsection{ANALYTICAL MODEL OF THE EARTH'S MAIN MAGNETIC FIELD}

The representation (21) may be useful in a description of the temporal variations $\dot{\bar{C}}_{n m}, \dot{\bar{S}}_{n m}$ of the Earth's gravitational potential with the aim of their permanent monitoring. However, at present we have several determinations of these values $(n=2,3,4)$ only on a very short time interval. Because the Earth's main magnetic field is time-dependent essentially, we can construct the corresponding analytical model of the kind (21) for the Earth's magnetic potential $\Phi(P, t)$, which traditionally may be represented in Gauss' form

$$
\Phi(P, t)=R \sum_{n=1}^{\infty}\left(\frac{R}{r}\right)^{n+1} \sum_{m=0}^{n}\left(g_{n m}(t) \cos m \lambda+h_{n m}(t) \sin m \lambda\right) \overline{\bar{P}}_{n}^{m}(\cos \vartheta),
$$

where $R$ is the mean radius of the Earth; $g_{n m}(t), h_{n m}(t)$ are Gauss' quasi-normalized harmonic coefficients and $\overline{\bar{P}}_{n}^{m}(\cos \vartheta)$ are the quasi-normalized by A. Schmidt associated Legandre functions.

Since the starting degree $n$ in the expression (26) is equal to $1(n>0)$, for this reason the function $\Phi(P, t)=\Phi(P, t=$ const $)$ may be considered as an element of the Hilbert space $H_{2}(\Sigma)$ of functions which are harmonic outside the Bjerhammar sphere, hence for every $t=$ const from $\Phi(P, t=$ const $) \in H_{2}(\Sigma)$ the expansion (26) is correct according to the Runge-Krarup theorem*).

It is evident also that Taylor's expansion of the Gauss' harmonic coefficients $g_{n m}(t), h_{n m}(t)$ (by neglecting terms of order two and higher) can be written

$$
\left.\begin{array}{l}
g_{n m}(t) \\
h_{n m}(t)
\end{array}\right\}=\left\{\begin{array}{l}
g_{n m} \\
h_{n m}
\end{array}\right\}+\left\{\begin{array}{l}
\dot{g}_{n m} \\
\dot{h}_{n m}
\end{array}\right\}\left(t-t_{0}\right),
$$

by analogy with the preceding expression (20). Then after the linear transformation (22) of the interval $[c, d]$ into the standard interval $[-1,1]$ (transport from time $t \in[c, d]$ to "standard time" $z \in[-1,1]$ ) by using the expressions (21), (23), (24), and (25) we get immediately the following representation

$$
\widetilde{\Phi}(P, z)=R \sum_{n=1}^{\infty}\left(\frac{R}{r}\right)^{n+1} \sum_{m=0}^{n}\left\{\sum_{k=0}^{\infty}\left(g_{n m}^{k} \cos m \lambda+h_{n m}^{k} \sin m \lambda\right) \cdot T_{k}(z)\right\} \overline{\bar{P}}_{n}^{m}(\cos \vartheta),
$$

of the function $\widetilde{\Phi}(P, z)$ instead of the function $\Phi(P, t)$ and

So then, we may write

$$
\left.\begin{array}{l}
\tilde{g}_{n m}(z) \\
\tilde{h}_{n m}(z)
\end{array}\right\}=\sum_{k=0}^{\infty}\left\{\begin{array}{l}
g_{n m}^{k} \\
h_{n m}^{k}
\end{array}\right\} T_{k}(z) \text {. }
$$

*) T. Krarup has gave in special discussion (Graz, 1996) a positive estimation of such applying Runge-Krarup theorem for a description of the time-dependent geomagnetic field (in the frame of approximate treatment). 
Table 2. Gauss' quasi-normalized coefficients of the static part of the geomagnetic field and their secular variations; reference epoch $t_{0}=1765.0 \mathrm{yr}$.

\begin{tabular}{|c|c|c|c|c|c|}
\hline$n$ & $M$ & $g_{n m},[$ in $\gamma]$ & $h_{n m},[$ in $\gamma]$ & $\dot{g}_{n m},[$ in $\gamma / \mathrm{yr}]$ & $\dot{h}_{n m},[\mathrm{in} \gamma / \mathrm{yr}]$ \\
\hline 1 & 0 & -33810 & 5046 & 16.4 & 22.3 \\
\hline 2 & 1 & -3496 & & 10.6 & -8.6 \\
& 0 & 579 & 802 & -4.7 & 23.9 \\
\hline 3 & 1 & -1229 & 604 & 17.7 & -2.1 \\
& 2 & 637 & -811 & 5.1 & 0.7 \\
& 0 & -269 & 268 & -2.6 & 3.9 \\
\hline 4 & 1 & 1286 & 937 & 5.6 & 3.7 \\
& 2 & -200 & & 4.8 & 5.2 \\
& 3 & 351 & 14 & -5.0 & -0.8 \\
& 1 & 836 & -492 & 0.2 & 2.5 \\
\hline
\end{tabular}

$$
\begin{aligned}
& \left.\begin{array}{l}
g_{n m} \\
h_{n m}
\end{array}\right\}=\sum_{k=0}^{\infty}(-1)^{k}\left\{\begin{array}{l}
g_{n m}^{2 k} \\
h_{n m}^{2 k}
\end{array}, \text { if } t_{0}=(d-c) / 2,\right. \\
& \left.\begin{array}{l}
\dot{g}_{n m} \\
\dot{h}_{n m}
\end{array}\right\}=\frac{2}{(d-c)} \sum_{k=1}^{\infty}(-1)^{k-1}(2 k-1)\left\{\begin{array}{l}
g_{n m}^{2 k-1} \\
h_{n m}^{2 k-1}
\end{array},\right.
\end{aligned}
$$

and note that the equations (27), (28), and (31) admit the next interpretation. On the one hand, by simple using Chebyshev's coefficients of the even degrees we may compute now by means of (30) Gauss' coefficients of the "static" or "permanent" part of the Earth's main magnetic field. On the other hand, the expression (31) provides the temporal variations in potential coefficients of the main magnetic field, which connected here with the certain sum (31) of Chebyshev's coefficients of the odd degrees.

Starting from the famous fundamental work (Gauss, 1838), the Earth's main magnetic field represents in Gauss' form (26), (27) for different reference epochs. The above formulae (28) - (31) provide the construction of one analytical model (28) of the Earth's main magnetic field on the ground of a preliminary defined time interval $t \in[c, d]$. Our first model was constructed for time interval [c=1845; d=1980] (Marchenko, 1998). In this study we have used 12 sets of harmonic coefficients $\left\{g_{n m}, h_{n m}\right\}$ for the following epochs: [c=1550 yr; 1600; 1650; $1700 ; 1750 ; 1800 ; 1845 ; 1900 ; 1922 ; 1945 ; 1965 ; \mathrm{d}=1980 \mathrm{yr}]$.

The approximation of each coefficient $g_{n m}(t)$ or $h_{n m}(t)$ was made by applying (22), (29) and leastsquares technique. Figure 1, Figure 2, and Figure 3 demonstrate some results of this closest approximation. Taking into account a preliminary nature of these investigations we decided to develop Chebyshev's expansions up to degree $k=6$ only for every coefficient $g_{n m}(t)$ or $h_{n m}(t)$ separately. As a result, the following truncations ( $1 \leq n \leq 4,0 \leq k \leq 6)$ of the constructed model (28) were derived and finally it consists of the coefficients $\left\{g_{n m}^{k}, h_{n m}^{k}\right\}$.

Next, using (30), (31), we get Gauss' coefficients of a permanent part of the geomagnetic field and their secular variations (Table 2 ) for the time interval $[c=1550 \mathrm{yr}, d=1980 \mathrm{yr}]$ with the reference epoch $\left(t_{0}=1765.0 \mathrm{yr}\right)$. The Figure 4 illustrates the declination of this "permanent part" of the Earth's magnetic field. Thus, we have considered, another way of looking at the description of the Earth's main magnetic and gravitational fields that caused by the following.

1. The uniform representation of the time-independent/time-dependent planet's fields in a certain finite interval of time. 
2. The possibility of a separation (in some generalized form) of the time-dependent and time-independent consistencies of the planet's potentials, which provides a simplification of direct problems (in a mathematically homogeneous form).

3. Simplest introducing the conventional reference epoch $t_{0}$ and the corresponding computation of the conventional time-independent "static" part of these potential fields for some epoch $t_{0}$.

\section{PLANET'S RADIAL DENSITY PROFILES}

Returning to the expression (1) for Newtonian potential, we can see that all problems regarding the determination of a planet's gravitational potential may be solved if the corresponding density functions $\rho\left(r^{\prime}, \vartheta^{\prime}, \lambda^{\prime}\right)$ are known. In practice we have another or inverse situation: we want to determine these functions (to take into account our poor knowledge about densities and some initial information about planets). First of all we want to determine the planet's radial densities $\rho\left(r^{\prime}\right)$ for instance, from the solution of inverse gravitational problem with an additional application of seismic data (Earth) and other geophysical data.

Recently, instead of the standard polynomial representation of a piecewise radial density some old hypotheses were analyzed especially in view of Williamson-Adams equation (Bullen, 1975; Marchenko, 1999; Marchenko, 2000). The latter leads to the special investigation of the hydrostatic/adiabatic Earth. Darwin's law (1884) of radial density was transformed to the so-called regular Darwin's law and further to the famous Gaussian distribution that was called the Earth's density normal law. Roche's density is treated as a truncated Taylor series expansion of Gauss' model (Marchenko, 2000; Marchenko, 2000a). In the frame of the hydrostatic/adiabatic planet a remarkable possibility was found additionally (Marchenko, 2000a): the density at the origin and seismic data at the boundaries of density jumps are sufficient to derive coefficients of piecewise Gauss' model (shell by shell).

Thus, the piecewise Gauss' radial profile can be based on the differential correction of created before Roche's model. In addition, the initial iteration for coefficients of Gauss' law can be provided by the seismic data alone at the boundaries of the Earth's jumps. The created radial profiles (continuous and piecewise) are in a good agreement with the PREM-density model, including gravity distribution inside the Earth. For this reason we decided to apply Gauss' normal model for the representation and comparison of planet's radial densities profiles.

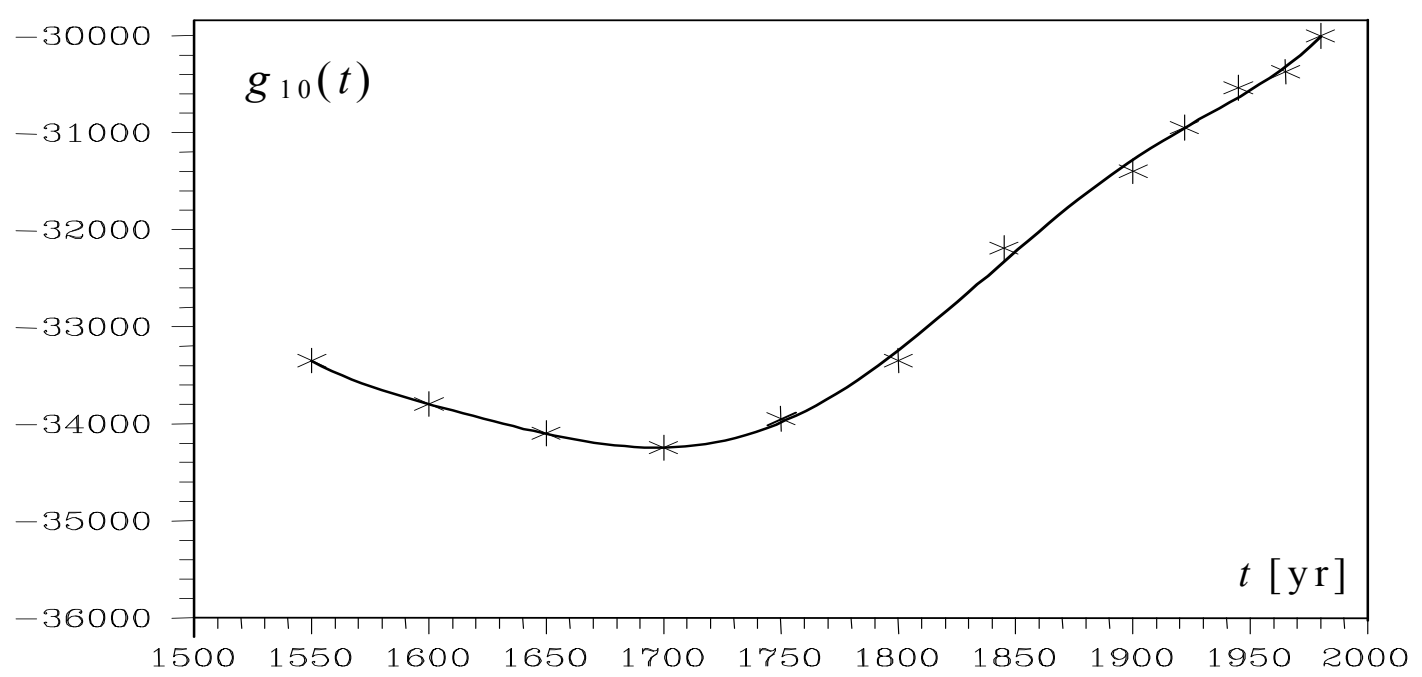

Figure 1. Chebyshev's approximation of the time-dependent coefficient $g_{10}(t)$ [in $\gamma$ ]. 


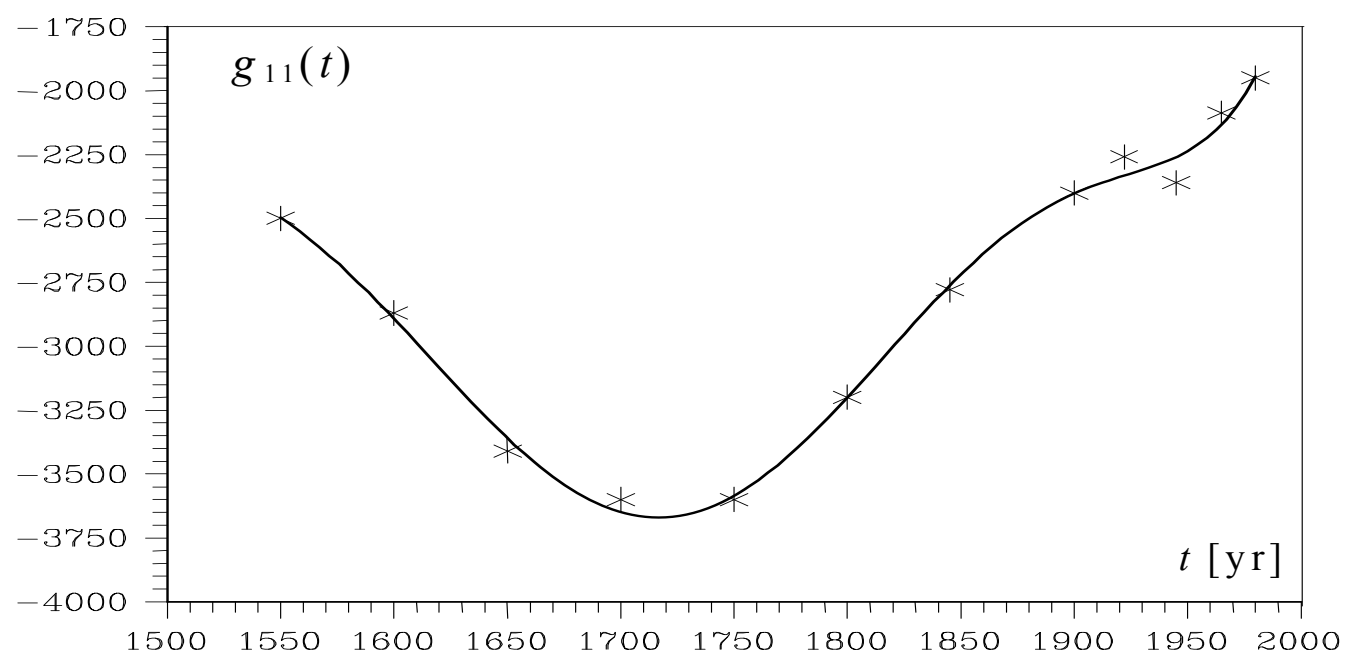

Figure 2. Chebyshev's approximation of the time-dependent coefficient $g_{11}(t)$ [in $\left.\gamma\right]$.

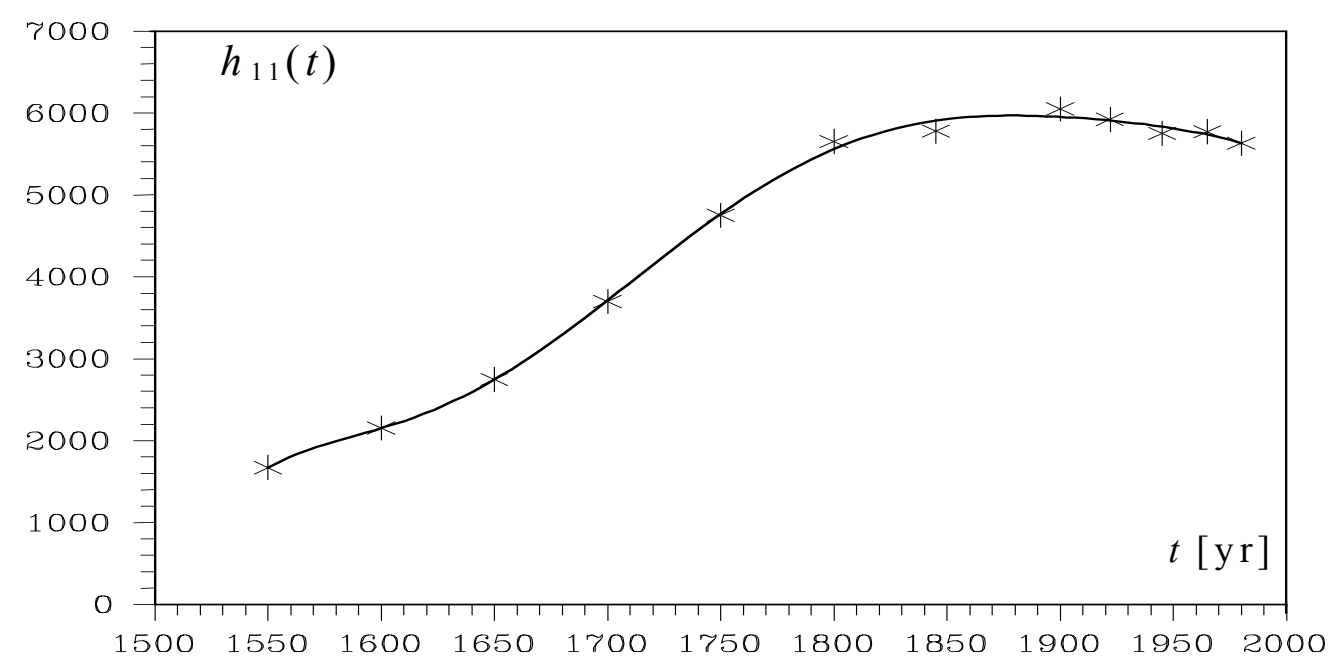

Figure 3. Chebyshev's approximation of the time-dependent coefficient $h_{11}(t)$ [in $\left.\gamma\right]$.

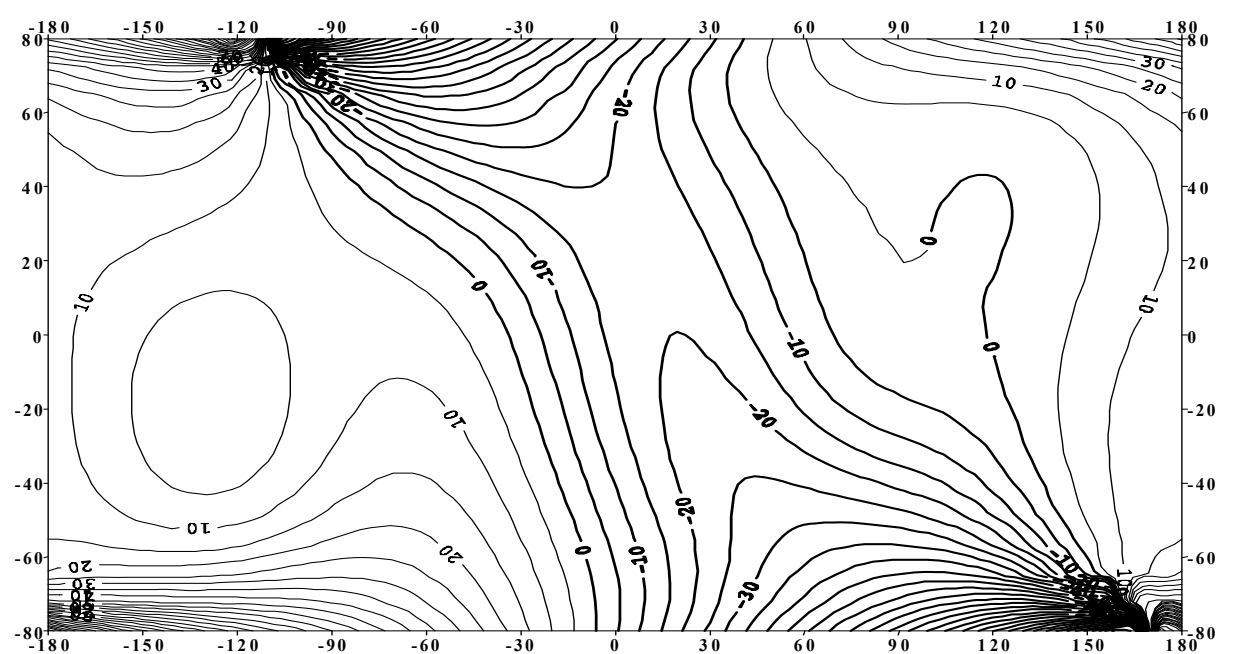

Figure 4. Declination [degree] of "static part" of the Earth's main magnetic field (epoch 1765.0). 


\subsection{STRATIFIED PLANET AND AUXILIARY RELATIONSHIPS}

First we shall assume the figure of a planet in form of a sphere with the certain mean radius $R$ (see, Table 4). In such a formulation the planet's radial density will be considered as a function $\rho(\ell)$ of one variable $\ell$ $(0 \leq \ell \leq R)$. Since we use a sphere instead an ellipsoid with the flattening $f=0$, the gravitational potential $V$ will be treated also as the gravity potential $W$ (Moritz, 1990).

The planet mass $M$ and its mean moment of inertia $I$ will be chosen as observed data. For latter use, we shall rewrite several well-known formulae in the case of a layered sphere of the radius $\ell$, restricted planet masses. If a stratification of the Earth leads to its division into $m$ shells, we shall represent a volume radial density by one model within every shell separately

$$
\rho_{i}(\ell), i=1,2, \ldots m .
$$

with a suitable mathematical representation of the functions (32). Then we shall consider these relationships for a spherical stratified Earth. Expression for the mass can be written now as

$$
M(\ell)=4 \pi \sum_{i=1}^{m-1} \int_{\ell_{i-1}}^{\ell_{i}} \rho_{i}(x) x^{2} d x+\int_{\ell_{m-1}}^{\ell} \rho_{m}(x) x^{2} d x,\left(\ell_{i-1}=0\right),
$$

and the mean moment of inertia will be represented in the similar way

$$
I(\ell)=\frac{8 \pi}{3}\left[\sum_{i=1}^{m-1} \int_{\ell_{i-1}}^{\ell_{i}} \rho_{i}(x) x^{4} d x+\int_{\ell_{m-1}}^{\ell} \rho_{m}(x) x^{4} d x\right],\left(\ell_{i-1}=0\right),
$$

where $d x$ is the element of a line. The mean density $D(\ell)$ and the gravity inside a planet admit the following representations

$$
\begin{gathered}
D(\ell)=\frac{3}{4 \cdot \pi \cdot \ell^{3}} M(\ell), \\
g(\ell)=\frac{4 \cdot \pi \cdot G}{3} \ell \cdot D(\ell) .
\end{gathered}
$$

In the case of the Earth we shall use the seismic velocities $V_{p}$ and $V_{s}$ as well-known function

$$
\Phi=\Phi(\ell)=V(\ell)_{P}^{2}-\frac{4}{3} V(\ell)_{S}^{2},
$$

by applying their grid values in accordance with the PREM-model (Dziewonski and Anderson, 1981) that reflect the results of seismic radial tomography of the Earth interior.

Assuming that such a piecewise density model must fulfill some basic differential equations, our nearest goal will consist of the computation of the integrals (33), (34) after the choice of an appropriate mathematical expression for the density distribution within each shell. The separation of the spherical planet into convenient shells has to be choice at those spheres, where discontinuities in the parameter $\Phi$ or in its derivative can be observed.

\subsection{AUXILIARY RELATIONSHIPS FOR GAUSS' DENSITY MODEL}

Note again (Marchenko, 2000a) that Gaussian distribution for radial density

$$
\rho(\ell)=\rho_{0} \exp \left(-\beta^{2} x^{2}\right),(\beta=\text { const }),
$$

represents a partial solution of Williamson-Adams equation

$$
\frac{d \ln \rho(\ell)}{d \ell}=-\frac{g(\ell)}{\Phi(\ell)} .
$$

where $x$ is the dimensionless "radius-vector" $x=\ell / R$ regarding to $R ; \rho_{0}=$ const and may be considered here as the density at the origin. So, instead of polynomials we come in (38) to the functional dependence for radial density as an exponential function. This dependence admits the next remarkable expressions for the mass and the 


$$
M(\ell)=\frac{4 \pi \rho_{0} R^{3}}{\beta^{2}}\left[\frac{\sqrt{\pi} \cdot \operatorname{erf}(\beta \cdot x)}{4 \beta}-\frac{x}{2 \rho_{0}} \rho(\ell)\right], I(\ell)=\frac{R^{2}}{\beta^{2}}\left[M(\ell)-\frac{4 \pi \ell^{3}}{3} \rho(\ell)\right],
$$

where $\operatorname{erf}(z)$ is the integral of the Gaussian distribution from 0 to $z$ or the probability integral with the normal density distribution according to (38).

If a suitable stratification of the Earth gives its division into $m$ shells, then we try to represent the density distribution by one Gauss' model within every shell separately

$$
\rho_{i}(\ell)=\alpha_{i} \exp \left(-\beta_{i}^{2} x^{2}\right), i=1,2, \ldots m .
$$

Inserting (41) into (33), (35), and (34) we get finally the recurrence formulae for computations of the mass, the mean density and the mean moment of inertia, respectively

$$
\begin{gathered}
M_{1, m}(\ell)=M_{1, m-1}\left(\ell_{m-1}\right)+M_{m}(\ell),\left(\ell_{m-1} \leq \ell \leq \ell_{m}\right), \\
D_{1, m}(\ell)=\left(\frac{\ell_{m-1}}{\ell}\right)^{3} D_{1, m-1}\left(\ell_{m-1}\right)+D_{m}(\ell), \\
I_{1, m}(\ell)=I_{1, m-1}\left(\ell_{m-1}\right)+I_{m}(\ell),
\end{gathered}
$$

where every integral in (33), (34) may be expressed by means of the auxiliary relationships

$$
\begin{gathered}
\psi_{i}\left(\ell_{i}\right)=\left[\frac{\sqrt{\pi} \cdot \operatorname{erf}\left(\beta_{i} \cdot x_{i}\right)}{4 \beta_{i}}-\frac{x_{i}}{2 \rho_{i-1}} \rho_{i}\left(\ell_{i}\right)\right], \\
M_{i}(\ell)=\frac{4 \pi \alpha_{i} R^{3}}{\beta_{i}^{2}}\left[\psi_{i}(\ell)-\psi_{i-1}\left(\ell_{i-1}\right)\right], M_{1,1}(\ell)=M_{1}(\ell), \\
D_{i}(\ell)=\frac{3 \alpha_{i} R^{3}}{\beta_{i}^{2} \ell_{i}^{3}}\left[\psi_{i}(\ell)-\psi_{i-1}\left(\ell_{i-1}\right)\right], D_{1,1}(\ell)=D_{1}(\ell), \\
I_{i}(\ell)=\frac{R^{2}}{\beta_{i}^{2}}\left\{\left[M_{i}(\ell)-\frac{4 \cdot \pi \cdot \ell^{3}}{3} \rho_{i}(\ell)\right]-\left[M_{i}\left(\ell_{i-1}\right)-\frac{4 \cdot \pi \cdot \ell_{i-1}^{3}}{3} \rho_{i}\left(\ell_{i-1}\right)\right]\right\}, I_{1,1}(\ell)=I_{1}(\ell),
\end{gathered}
$$

starting from the first shell $\left(0 \leq \ell \leq \ell_{1}\right)$. In these formulae $\ell_{j}(j=1,2, \ldots m-1)$ are the fixed radius-vectors, where discontinuous of the radial density are presupposed. The recurrence formula for gravity is based on the expressions (36) and (43):

$$
g_{1, m}(\ell)=\frac{4 \cdot \pi \cdot G}{3} \ell \cdot D_{1, m}(\ell),\left(\ell_{m-1} \leq \ell \leq \ell_{m}\right) .
$$

For the recurrence formulae of the seismic parameter $\Phi$ and its jumps we shall find

$$
\frac{d \ln \rho_{i}(\ell)}{d \ell}=-2 \beta_{i} \frac{\ell}{R^{2}}
$$

Further by applying (50) and Williamson-Adams equation (39) for the piecewise model (41) after some manipulations we get

$$
\begin{gathered}
\Phi_{1, m}(\ell)=\left(\frac{\beta_{m-1}^{2}}{\beta_{m}^{2}}\right) \cdot\left(\frac{\ell_{m-1}}{\ell}\right) \cdot \Phi_{1, m-1}\left(\ell_{m-1}\right)+\Phi_{m}(\ell)=\frac{G M_{1, m}(\ell) \cdot R^{2}}{2 \beta_{m}^{2} \ell^{3}},\left(\ell_{m-1} \leq \ell \leq \ell_{m}\right), \\
\Phi_{i}(\ell)=-\frac{2 \cdot \pi \cdot G \cdot R^{5}}{\beta_{i}^{4} \ell^{3}} \alpha_{i}\left[\psi_{i}(\ell)-\psi_{i}\left(\ell_{i-1}\right)\right], \Phi_{1,1}(\ell)=\Phi_{1}(\ell),\left(0 \leq \ell \leq \ell_{1}\right) .
\end{gathered}
$$

By the definition (37) of the parameter $\Phi$ we come to the remarkable ratio

$$
\frac{\Phi_{1, i}\left(\ell_{j}\right)}{\Phi_{1, i+1}\left(\ell_{j}\right)}=\frac{\beta_{i+1}^{2}}{\beta_{i}^{2}}>0, i=1,2, \ldots m
$$

which must be positive for each boundary of two shells. 
Table 3. The Earth's piecewise Gauss' density model ( $m=7$ )

\begin{tabular}{|c|c|c|c|c|c|}
\hline Shell & $\alpha_{i}$ & Initial iteration $\beta_{i}$ & Final iteration $\beta_{i}$ & $\ell_{j}, \mathrm{~km}$ & Density jump \\
\hline 1 & 13.061 & 0.8248 & 0.8280 & 1221.5 & 0.587 \\
2 & 12.483 & 0.8171 & 0.8611 & 3480.0 & 4.465 \\
3 & 6.370 & 0.6100 & 0.6772 & 5701.0 & 0.392 \\
4 & 6.058 & 0.6308 & 0.7165 & 5971.0 & 0.329 \\
5 & 5.784 & 0.6437 & 0.7523 & 6151.0 & 0.084 \\
6 & 6.057 & 0.6984 & 0.8066 & 6346.6 & 0.520 \\
\hline
\end{tabular}

Finally we can compute the seismic jump of $\Phi$ at the $j$ - boundary as

$$
\Delta \Phi=\Delta \Phi_{i, i+1}=\Phi_{i}\left(\ell_{j}\right)-\Phi_{i+1}\left(\ell_{j}\right)=\frac{G \cdot M_{1, i}\left(\ell_{j}\right) \cdot R^{2}}{2 \ell_{j}^{3}}\left[\frac{1}{\beta_{i}^{2}}-\frac{1}{\beta_{i+1}^{2}}\right] .
$$

This formula may be used also as the additional condition between the coefficients of every shell, because the left-hand side of (54) is known from seismic data. Auxiliary relationships of the same type for piecewise Roche's model can be found in (Marchenko, 1999).

\subsection{THE EARTH'S PIECEWISE GAUSS' MODEL}

Further a process of the differential correction for the creation of piecewise Gauss' model was applied. The density at the origin according to Gauss' model is depended on the observed $\Phi$ as

$$
\Phi_{1}(0)=-\frac{2 \cdot \pi \cdot G \cdot R^{2}}{3 \cdot \beta_{1}^{2}} \rho_{0}
$$

where $\Phi_{1}(0)$ corresponds to the first piece of the seismic data $\Phi$ at the origin. According to the stratification of (Marchenko, 1999) we get $i=1,2, \ldots m=7$ pieces (see Table 3). Next, taking into account (53) and (55), after simple manipulations the following formula may be written

$$
\beta_{m}=-\frac{2 \pi G R^{2} \rho_{0}}{3 \Phi_{1}(0)} \cdot \frac{\Phi_{1}\left(\ell_{1}\right) \cdot \Phi_{2}\left(\ell_{2}\right) \cdot \Phi_{3}\left(\ell_{3}\right) \ldots \Phi_{m-1}\left(\ell_{m-1}\right)}{\Phi_{2}\left(\ell_{1}\right) \cdot \Phi_{3}\left(\ell_{2}\right) \cdot \Phi_{4}\left(\ell_{3}\right) \ldots \Phi_{m}\left(\ell_{m-1}\right)}
$$

which leads to a new remarkable conclusion:

If a planet density is known at the origin, the seismic data $\Phi$ at the boundaries of a planet jumps are sufficient for the independent determination of the coefficients (56) of the piecewise Gauss' model.

Thus, the formula (56) can be applied to the computation of these coefficients $\beta_{i}$. (It is evident, that the same approach may be used for their determination, starting from the density at the Earth's surface). In this paper we shall use the equality

$$
\alpha_{i}=a_{i}
$$

for the computation of the coefficients $\alpha_{i}$ of the piecewise Gauss' model, which were adopted on this step as the fixed values according to the previous Roche's model (Marchenko, 2000).

In fact, the coefficients $\beta_{i}$ corresponded to (56) and (57) yield the Gaussian density denoted below (see, Figure 5, Table 3) as "initial iteration" for such model. The final version of Gauss' radial density is denoted in the Figure 5 and Table 3 as "final iteration". This model was constructed by means of the linearization of necessary equations and direct approximation of the observed seismic data $\Phi$ in accordance with (Dziewonski and Andeson, 1981).

Solution of the mentioned non-linear inverse problem was based on the expressions (41)-(54) and 3 additional conditions for the Earth's mean density, the mean moment of inertia, and the surface density, which was adopted here as for the piecewise Roche's model: $\rho_{s}=2.67 \mathrm{~g} / \mathrm{cm}^{3}$. Differences in the Table 3 between the initial and final versions of the coefficients $\beta_{i}$ of Gauss' models may be treated for every pieces by the wellknown fact: the Earth's radial density satisfies to Williamson-Adams equation better and better with decreasing of the parameter $\ell$. 
Table 4. Fundamental astronomical and geodetic constants of the planets

\begin{tabular}{|l|ccccc|cccc|}
\hline Planet & Mercury & Venus & Earth & Moon & Mars & Jupiter & Saturn & Uranus & Neptune \\
\hline Radius $(\mathrm{km})$ & 2439 & 6052 & 6371 & 1738 & 3390 & 71398 & 60000 & 26145 & 24700 \\
Mean density $\left(\mathrm{g} / \mathrm{cm}^{3}\right)$ & 5.44 & 5.25 & 5.514 & 3.344 & 3.94 & 1.334 & 0.69 & 1.26 & 1.67 \\
Mean moment of inertia & $0.324^{*}$ & $0.334^{*}$ & 0.3298 & 0.3957 & 0.364 & $0.28^{*}$ & $0.26^{*}$ & $0.27^{*}$ & $0.27^{*}$ \\
Surface density $(\mathrm{g} / \mathrm{cm} 3)$ & 3.3 & 2.8 & 2.67 & 3.08 & 2.7 & 0.35 & 0.36 & 0.3 & 0.3 \\
\hline
\end{tabular}

\subsection{THE PLANET'S RADIAL DENSITY DISTRIBUTIONS}

Finally we shall characterize some properties of the planet's radial density profiles on the ground of the continuous Gauss' density distribution (as one shell) and come back again to three standard conditions listed in (Moritz, 1990). After the transformation of (40) to the dimensionless moment of inertia $I_{d}=I / M R^{2}$ we get the simplest relationship

$$
I_{d}=\frac{1}{\beta^{2}}\left[1-\frac{\rho_{s}}{D}\right],
$$

between the planet's mean density $D$, the moment of inertia $I_{d}$, and the surface density $\rho_{s}$. This formula provides for our non-linear inverse problem the remarkable closed expression

$$
\beta^{2}=\frac{1}{I_{d}}\left[1-\frac{\rho_{s}}{D}\right],
$$

for the determination of the coefficient $\beta$ of continuous Gauss' density model.

Thus, we come to the sequential solution of the non-linear inverse problem by means of two closed expressions for 2 basic parameters. The first one is the qualitative characteristic (59) of the global density distribution, which based on our knowledge of the planet mean density, the mean moment of inertia, and density on the planet' surface. For instance, this parameter for the Earth will be equal to $\beta=1.25022$ (if initial data are taken from the Table 4).

The second one is the quantitative characteristic

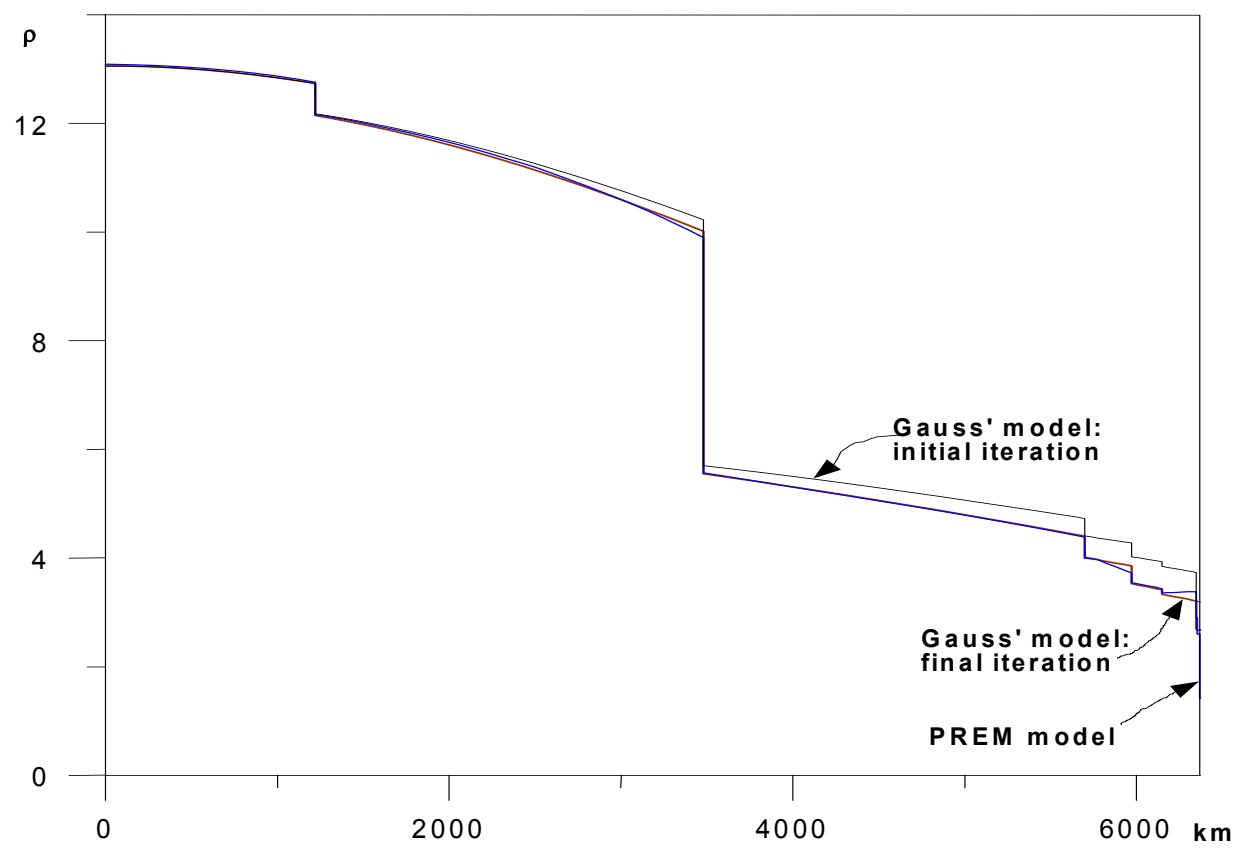

Figure 5. Comparison of the PREM-density $\rho\left[\mathrm{g} / \mathrm{cm}^{3}\right]$ between initial and final Gauss models.

\footnotetext{
* The sign ${ }^{*}$ denotes the computation of the planet's mean moment of inertia on the basis of Radau - Darwin theory.
} 


$$
\rho_{0}=\frac{4 \cdot \beta^{3} D \cdot \exp \left(\beta^{2}\right)}{3 \cdot\left(\sqrt{\pi} \cdot \exp \left(\beta^{2}\right) \cdot \operatorname{erf}(\beta)-2 \beta\right)},
$$

which was derived on the ground of the expression (47) for Gauss' mean density. Note that the determination of 2 parameters of the continuous Roche's model is based traditionally on the mean density $D$ and the mean moment of inertia $I_{d}$. As a matter of fact, in the case of the continuous Gauss' density model with 2 parameters all these 3 conditions can be replaced by one relationship (59) for the qualitative characteristic $\beta$ of the planet's normal density distribution. The quantitative characteristic $\rho_{o}$ now is the non-linear function (60) of the computed $\beta$ and the planet' mean density.

The solution of (59)-(60) provides finally the density at the centre of the Earth mass $\rho_{\mathrm{o}}=12.919 \mathrm{~g} / \mathrm{cm}^{3}$, and a remarkable agreement of $\rho_{s}=2.67 \mathrm{~g} / \mathrm{cm}^{3}$, the mean density $D$ and the mean moment of inertia $I_{d}$. Such model is in a good agreement with the continuous Bullard's model (which has 3 parameters) and its represents best of all the global trend of the piecewise PREM-model. This fact leads to the idea to apply Gaussian distribution of density for another planets, where seismic data for the stratification or tomography of a planet interior are absent.

Our initial information about the mean density, mean moment of inertia, and surface density are collected in the Table 4 and based on determinations of planet's masses, in particular, by means of different satellite and spacecraft programs. A main part of these data about numerical values of mean moment of inertia was computed on the basis of Radau - Darwin theory, (Bullen, 1975; Moritz, 1990, etc.), because observed information is doubtful or absent.

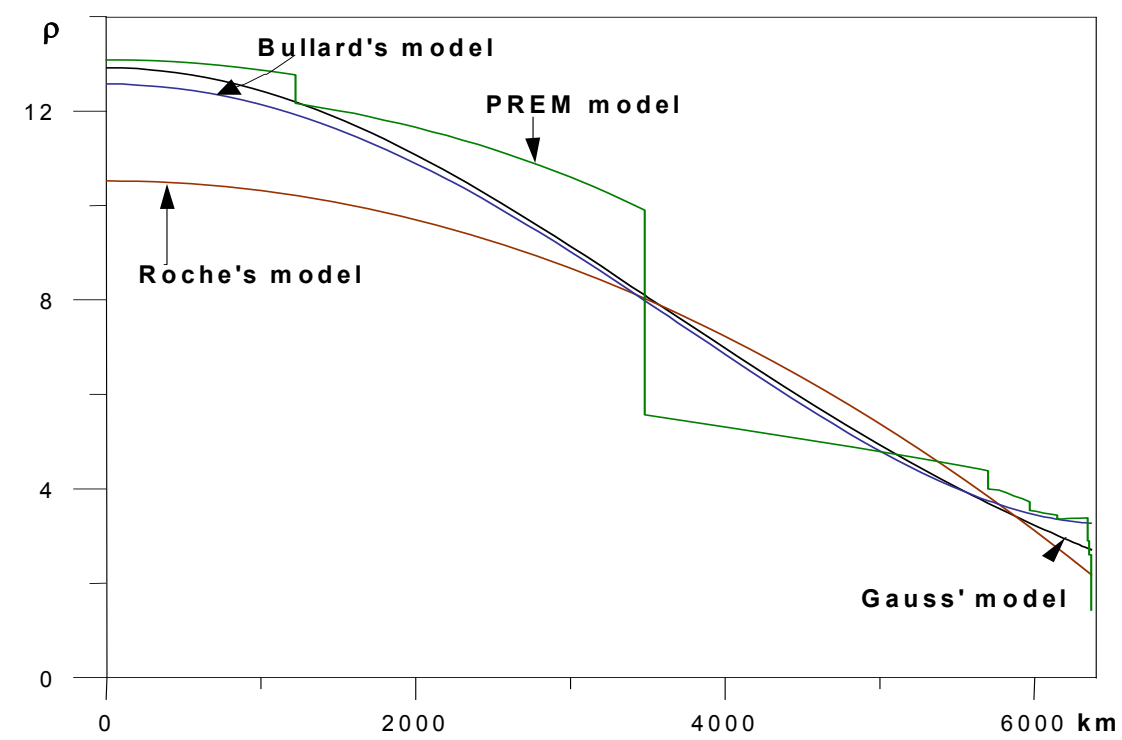

Figure 6. The Earth's radial density profiles regarding the piecewise PREM-density $\rho\left[\mathrm{g} / \mathrm{cm}^{3}\right]$ 
Table 5. Basic parameters of Roche and Gauss models of the planets radial density

\begin{tabular}{|c|c|c|c|c|c|}
\hline Planet & $A\left(\mathrm{~g} / \mathrm{cm}^{3}\right)$ & $b\left(\mathrm{~g} / \mathrm{cm}^{3}\right)$ & $\rho_{\mathrm{s}}=a+b\left(\mathrm{~g} / \mathrm{cm}^{3}\right)$ & $\beta$ & $\rho_{o}\left(\mathrm{~g} / \mathrm{cm}^{3}\right)$ \\
\hline Mercury & 10.866 & -9.044 & 1.82 & 1.102 & 10.701 \\
\hline Venus & 9.798 & -7.580 & 2.22 & 1.182 & 11.332 \\
\hline Earth & 10.582 & -8.446 & 2.14 & 1.250 & 12.919 \\
\hline Moon & 3.531 & -0.312 & 3.22 & 0.447 & 3.764 \\
\hline Mars & 5.841 & -3.124 & 2.72 & 0.937 & 6.537 \\
\hline Jupiter & 3.435 & -3.501 & $-0.07 \rightarrow ?$ & 1.623 & 5.067 \\
\hline Saturn & 1.958 & -2.113 & $-0.16 \rightarrow ?$ & 1.356 & 1.845 \\
\hline Uranus & 3.410 & -3.593 & $-0.17 \rightarrow ?$ & 1.680 & 5.166 \\
\hline Neptune & 4.519 & -4.749 & $-0.23 \rightarrow ?$ & 1.743 & 7.458 \\
\hline
\end{tabular}
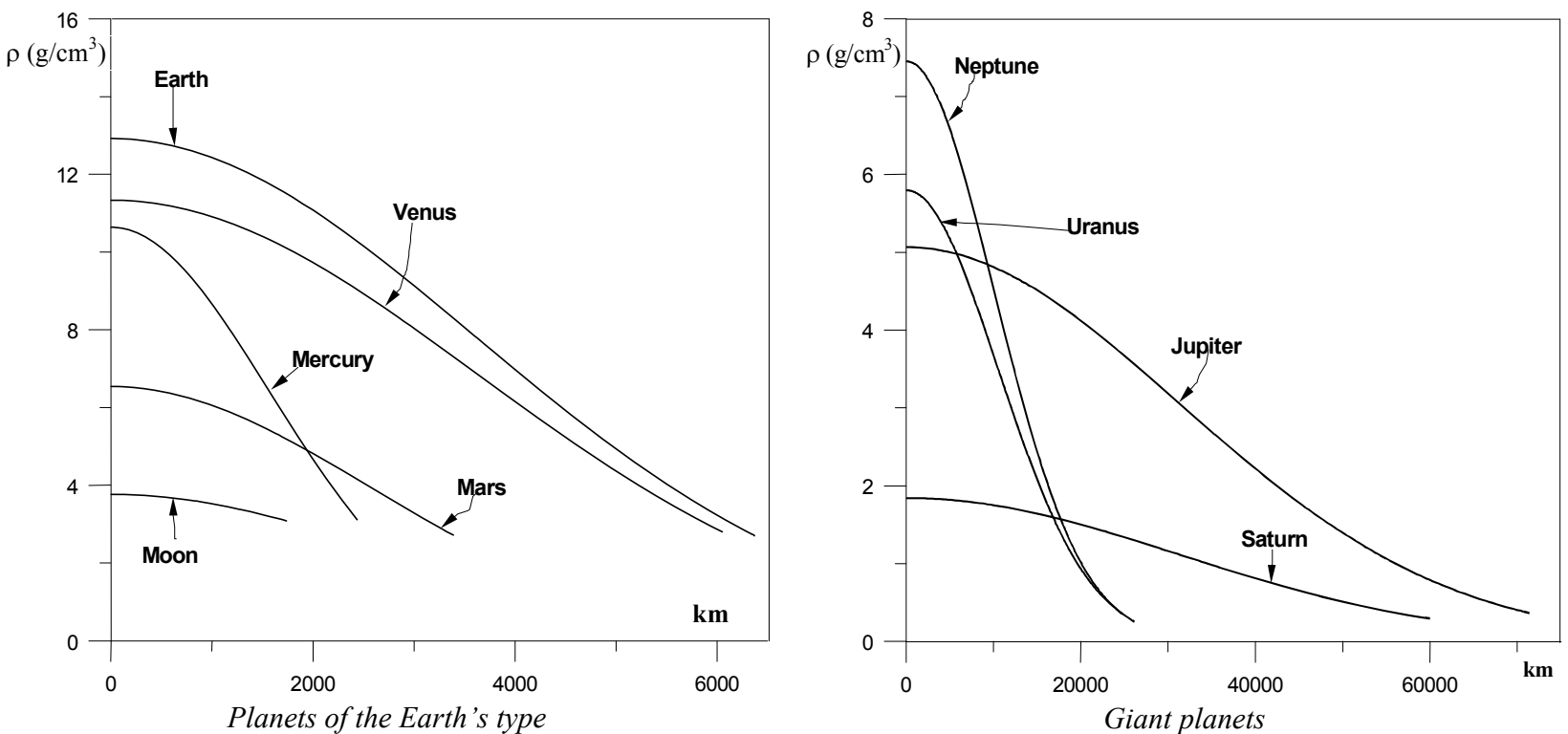

Figure 7. Gauss' radial density distributions inside the Moon and the planets
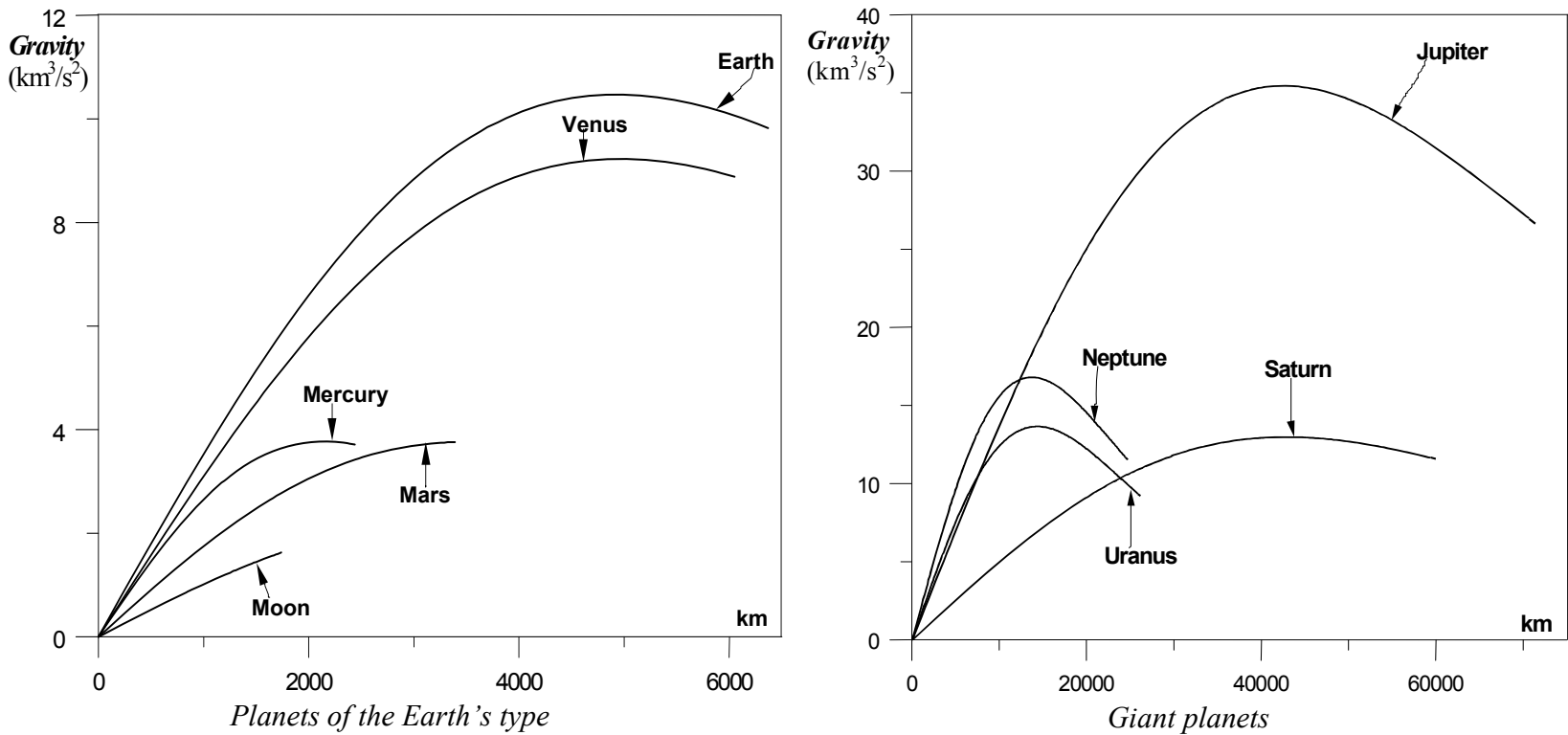

Figure 8. Gravity inside the planets according to the gaussian distributions 
Because a surface density may be considered as most unreliable parameter we decided to build the corresponding continuous Roche's models, based on the mean density and the mean moment of inertia only with further computations of Roche's surface density (Table 5). The examples of the Earth and Venus (observed $\rho_{\mathrm{s}}$ ) lead to the conclusion that Roche's surface density is rather smaller (excluding the Moon) than observed values near $0.5-0.6 \mathrm{~g} / \mathrm{cm}^{3}$. This fact was used as additional information for the creation of Gauss' models. For instance, the case of giant planets leads, in accordance with Roche's models, to the negative surface densities and some additional geophysics data were chosen for all such doubtful information (Bullen, 1975).

The results of the creation of the general Gaussian trend of density distributions can be found in Table 5 and Figure 7. Figure 8 reflects the gravity distributions inside the planets, which are corresponded these Gaussian density profiles. Finally we note that all continuous models are in a good agreement of well-known piecewise density planets models and for this reason they can be considered as some "ideal" radial densities in the frame of hydrostatic/adiabatic theory. The reader is invited to analyse these results.

1. Bullen, K. E. (1975) The Earth's Density. Chapman and Hall, London.

2. Bursa, M. (1992). Parameters of common relevance of astronomy, geodesy and geodynamics. The Geodesist's Handbook (ed. C.C.Tscherning), 193-197, Springer International.

3. Cheng, M.K., Eanes, R.J., Shum, C.K., Shutz, B.E., Tapley, B.D. (1989). Temporal variations in low degree zonal harmonic from Starlette orbit analysis. Geophysical Res. Letters, Vol. 16, No 5, 393-396.

4. Darwin, G. (1884) On the figure of equilibrium of a planet of heterogeneous density. Proceeding of the Royal Society, Vol. XXXVI, 158-166.

5. Dziewonski, A.M. and Anderson, D.L. (1981) Preliminary reference Earth model. Physics of the Earth and Planetary Interiors, Vol. 25, 297-356.

6. Groten E. (1999) Report of Special Commission 3 on „Fundamental Constants“ (SCFC). Paper presented at the General Assembly of IUGG, (Birmingham, 1999).

7. Heck, B. (1987). Time dependent geodetic boundary value problems. Proceed. of the International Symposium "Figure and Dynamics of the Earth, Moon and Planets", Prague, Monogr. Ser. VUGTK, 195-225.

8. IERS Standards (1989). IERS Technical Note, Central Bureau of IERS, Observatoire de Paris, No 3, 76 p.

9. Krarup, T. (1969). A Contribution to the Mathematical Foundation of Physical Geodesy. Danish Geod. Inst. Public., No 44, Copenhagen.

10. Marchenko, A.N. (1998) Parameterization of the Earth's gravity field. Point and line singularity. Lviv Astronomical and Geodetic Society, Lviv, 1998.

11. Marchenko, A.N. (1999) Simplest solutions of Clairaut's equation and the Earth's density model. In: "Quo vadis geodesia...?" (F.Krumm and V.S. Schwarze, Eds.). Universität Stuttgart, Techn. Reports Depart. of Geodesy and Geoinformatics, Report Nr. 1999. 6-2, 303-311.

12. Marchenko, A.N. (2000) Solutions of Clairaut's equation and the piecewise Roche's density model. Astronomical School's Report. Uman University, Vol. 1, No 1, 34-43.

13. Marchenko, A.N. (2000a) Earth's radial density based on Gauss' model. Paper presented at the conference "Modern Achievements of Geodesy”. (April, 2000, Lviv), University of Lviv, 2000, 18-26.

14. Marchenko A.N., Abrikosov O.A. (1999) Evolution of the Earth's Principal axes and Moments of Inertia: The Canonical Form of Solution. In: Proceedings of the second International Symposium "Geodynamics of the Alps-Adria Area by means of Terrestrial and Satellite Methods”, Dubrovnik, CROATIA, 1998 (K. Čolič and H. Moritz, Eds.). Zagreb and Graz, 1999, 177 - 202.

15. Moritz, H. (1980). Advanced Physical Geodesy, Wichmann, Karlsruhe, 1980.

16. Moritz, H. (1990) The Figure of the Earth. Theoretical Geodesy and Earth's Interior, Wichmann, Karlsruhe, 1990.

17. Moritz, H., and Mueller, I.I. (1987). Earth's Rotation. Theory and Observation. New York, Ungar.

18. Maxwell, J.K. (1881). A Treatise on Electricity and Magnetism. 2nd Edition, Oxford, Vol.1, 179-214.

19. Neyman, Yr. (1979). Variational method of Physical Geodesy, Nedra, Moscow, (in Russian)

20. Rubincam, D.P. (1984). Postglacial rebound observed by LAGEOS and effective viscosity of the lower mantle. $J$. Geophys. Res., vol.89, 1077-1087.

21. Sacerdote, F., and Sanso, F. (1987). New developments of boundary value problems in Physical Geodesy. Dipartimento di Mathematica Universita di Pisa, Public. No 208, Pisa.

22. Yoder, C.F., Williams, J.G., Dickey, J.O., Schutz, B.E., Eanes, R.J., Tapley, B.D. (1983). Secular variation of Earth's gravitational harmonic $\mathrm{J}_{2}$ coefficient from LAGEOS and nontidal acceleration of Earth rotation. Nature, vol.303, 757762.

Received 20.05.2000 